\title{
Left Bundle Branch Block in Suspected Acute Myocardial Infarction: to Early Reperfuse or Not?
}

\author{
Gabrielle Alexander Kartawan
}

Faculty of Medicine, Udayana University

\begin{tabular}{|c|c|}
\hline ARTICLE INFO & A B S T R A C T \\
\hline $\begin{array}{l}\text { Keyword: } \\
\text { Left Bundle Branch Block } \\
\text { Acute Myocardial Infarction } \\
\text { STEMI } \\
\text { Early reperfusion } \\
\text { Sgarbossa }\end{array}$ & $\begin{array}{l}\text { Diagnosing Acute Myocardial Infarction (AMI) in the setting of the Left } \\
\text { Bundle Branch Block (LBBB) proposes a great challenge to the clinician. } \\
\text { Despite current therapy recommendations, early reperfusion may not be } \\
\text { appropriate for all patients with new or presumed new LBBB because only a } \\
\text { minority is caused by acute myocardial infarction with true occluded artery. } \\
\text { This is a case of a } 47 \text {-year-old man presented at a rural hospital emergency } \\
\text { with highly suspected acute myocardial infarction, a history of old infarct, } \\
\text { and LBBB on ECG. Relying on careful clinical examination and close } \\
\text { evaluation of hemodynamic with serial ECG, the patient was decided to be } \\
\text { managed conservatively. Thorough clinical judgment is required in } \\
\text { diagnosing and managing patients with acute MI and LBBB. Avoiding } \\
\text { unnecessary burdens and risks resulting from early reperfusion therapy } \\
\text { may have a significant impact especially in the centre with limited modality } \\
\text { settings. }\end{array}$ \\
\hline
\end{tabular}

*) corresponding author

Faculty of Medicine, Udayana University

Email: gabriellekartawan@gmail.com

DOI: $10.47679 /$ makein.202135

This open access article is under the CC-BY-SA license.

\section{INTRODUCTION}

LBBB often alter the ventricular depolarization which can mask the ST/T wave changes and eliminate pathologic Q wave which usually localizes infarct area in ECG for acute myocardial infarction (AMI). ESC STEMI Guidelines (2017) suggested using Sgarbossa's 3 ECG criteria in diagnosing myocardial infarction in the setting of LBBB. The criteria include: 1) Concordant ST-segment elevation of $\geq 1 \mathrm{~mm}$ in the presence of a positive QRS complex, 2) ST-segment depression of $\geq 1 \mathrm{~mm}$ in lead $\mathrm{V} 1, \mathrm{~V} 2$, or $\mathrm{V} 3$, and 3 ) Discordant ST-segment elevation with ST/S ratio of $\leq-0.25$. All measurements are made from the PR segment and STsegment is measured from the J-point. These criteria have an overall sensitivity of $91 \%$ and specificity of $90 \%$ (Smith et al., 2012).

Meanwhile, current guidelines recommend that patients with likely myocardial infarction (MI) who have new or presumed new LBBB is considered diagnostic of AMI and should undergo early reperfusion therapy. Despite this recommendation, early reperfusion may not be appropriate for all patients with new LBBB because only a minority is diagnosed with myocardial infarction (Kontos et al., 2011). The prevalence of false-positive catheterization laboratory activation is frequent among patients with LBBB and a significant proportion of LBBB patients with AMI do not have an occluded culprit artery in catheterization. (Neeland et al., 2012). In facts, true acute MI-associated LBBB is rare but not performing adequate reperfusion therapy to these group are related to a high risk of congestive heart failure and death (Wahab, 2017). These considerations create a dilemmatic decision in diagnosing and managing patients with LBBB and suspected AMI.

The implications are even more challenging in centers that do not have immediate access to early reperfusion therapy, where false-positive fibrinolytic or catheterization activation would impose a burden in the referral process, unnecessary risks, and on the other hand resulting in huge expenses. Here we present a case of a patient with typical angina and highly suspected AMI with history of old infarct and LBBB on ECG at the rural hospital where careful clinical judgment is required to more precisely diagnose and manage these patients even in the very limited clinical settings. 


\section{CASE REPORT}

Male, 47 years old, came to the emergency department with chest pain for approximately 12 hours before admission. The pain was described as heavy, radiating to the back, getting worse with activity, and occurred for more than 20 minutes each episode. He said that the pain was initially felt approximately 6 days ago but immediately disappeared after some rest. He denied any shortness of breath. The patient acknowledged that there was a history of the same chest pain before, approximately 2 years ago. He was hospitalized around 3 days back then but denied any fibrinolytic or PCI procedure. He wasn't sure about the diagnosis of his chest pain back then but after hospitalization, he had not been taking any routine medication or appointment until the present. The patient denied other relevant past medical history. He is a nonsmoker and has no family history of coronary artery disease or sudden cardiac death.

At presentation, he was afebrile, blood pressure was $130 / 75$, heart rate was $55 \mathrm{bpm}$, respiratory rate was 18 per minute, and the peripheral oxygen saturation was $99 \%$ on room air. Physical examination was unremarkable. A 12lead-ECG revealed a sinus rhythm with a rate of $52 \mathrm{bpm}$ (Figure 1). There was a prolonged QRS interval in the lateral precordial lead, a dominant $\mathrm{S}$ wave in $\mathrm{V} 1$, and pathology $\mathrm{Q}$ waves in the inferior lead, suggesting LBBB and Inferior Old Myocardial Infarction. Smith's Modified Sgarbossa criteria for diagnosing AMI in the setting of LBBB were all negative in this initial ECG.

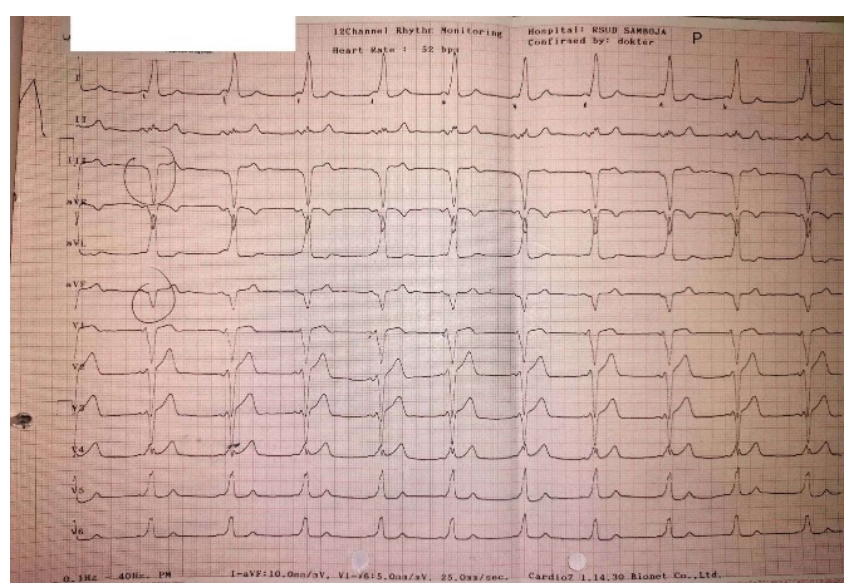

Figure 1. Initial ECG at presentation

Laboratory findings and Chest X-Ray were unremarkable. There was no sign of cardiac enlargement. Examination of cardiac biomarkers, echocardiography, and angiography was not available in our center. Our center is also a non-PCI and non-fibrinolytic center. The patient was initially given a loading dose of $320 \mathrm{mg}$ of aspirin, a loading dose of $300 \mathrm{mg}$ of clopidogrel, $5 \mathrm{mg}$ of isosorbide dinitrate sublingually, and was planned for serial ECG. The patient was decided for a conservative strategy while closely being monitored. Other treatments were a maintenance dose of aspirin $80 \mathrm{mg}$ once daily, a maintenance dose of clopidogrel $75 \mathrm{mg}$ once daily, isosorbide dinitrate $5 \mathrm{mg}$ thrice daily or when chest pain reoccurred, laxative, simvastatin $30 \mathrm{mg}$ once daily, and candesartan $4 \mathrm{mg}$ twice daily.

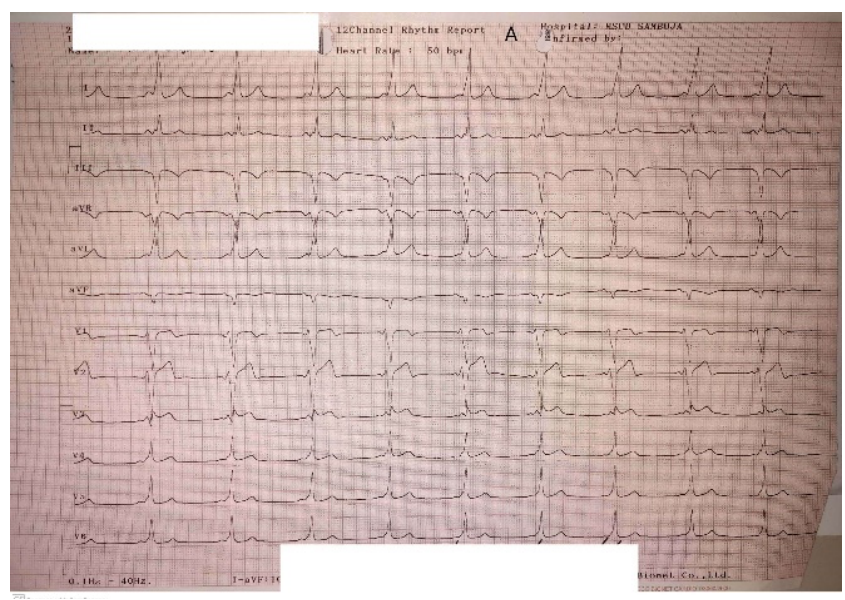

Figure 2. First serial ECG taken 12 hours after initial ECG

Serial ECG was done 12 hours after the initial ECG (Figure 2 ). There was concordant ST-Elevation for $2 \mathrm{~mm}$ in precordial lead V3. Interestingly, in this second ECG, the morphology rhythm of the V3 is quite different compared to the V3 in the initial ECG. Considering the clinical evaluation, the chest pain had already resolved and there were no signs of pulmonary edema from heart failure in physical examination, we suggested that there was a variation in precordial lead placement between the first and serial ECG and concluded that the concordant ST-Elevation was not a "STEMI equivalent" new LBBB. The patient was diagnosed with Non-ST Elevation Acute Coronary Syndrome and categorized as low risk based on ESC STEMI Guideline. Conservative strategy was continued with additional treatment of Fondaparinux $2.5 \mathrm{mg}$ subcutaneously for 5 days. The following day ECGs didn't show any ST Elevation (Figure 3 \& Figure 4). The patient remained stable throughout the hospital stay and was discharged on the sixth day. Referral for further evaluation and elective coronary angiography was planned via Cardiovascular Policlinic at the higher facilitated hospital.

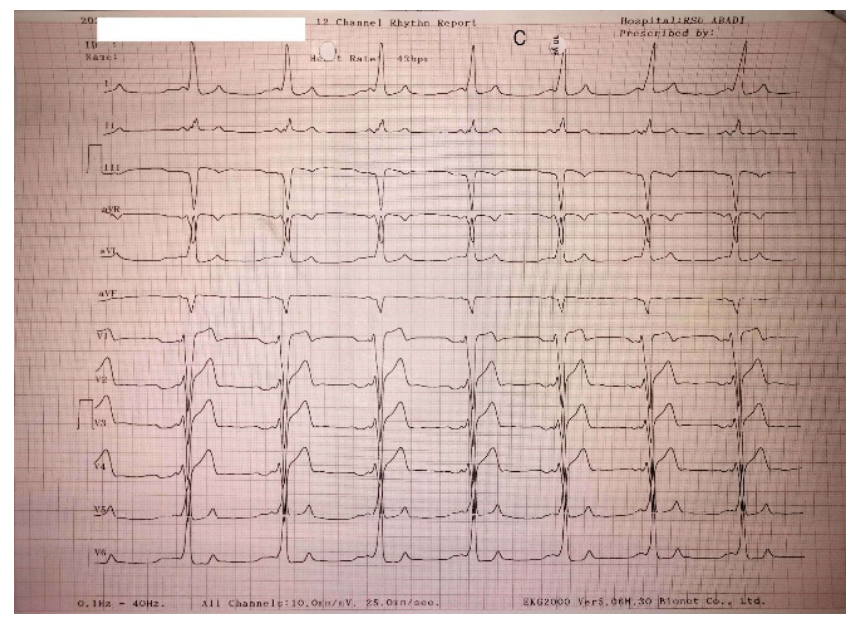

Figure 3. ECG taken on third day 


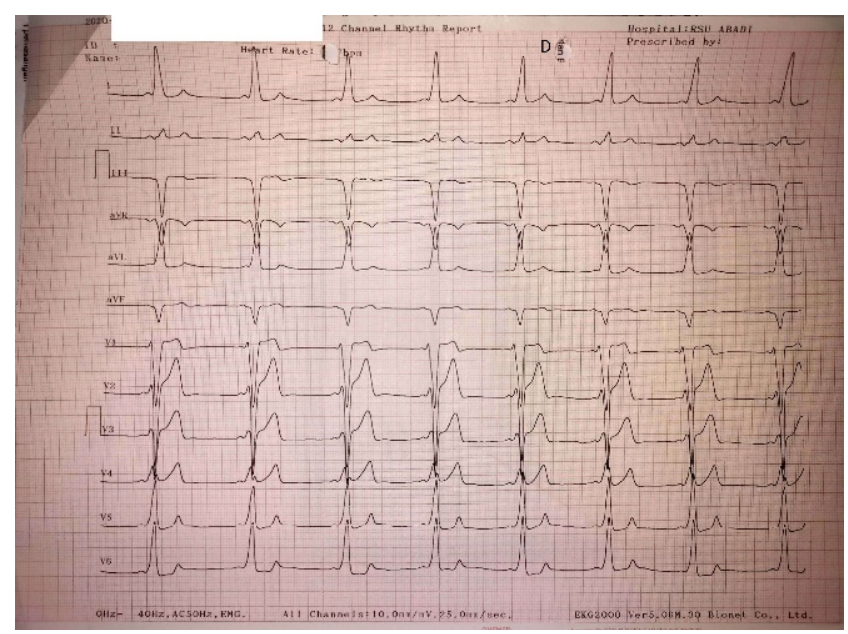

Figure 4. ECG on fourth day

\section{DISCUSSION}

Left bundle branch block is one of the conduction tissue diseases caused by delayed or block conduction within $\geq 1$ of the branches of the His-Purkinje system resulting in QRS abnormalities. In adults, LBBB defined as (Kusumoto et al., 2018):

1. QRS duration $\geq 120 \mathrm{~ms}$

2. Broad notched or slurred $R$ wave in leads $I, a V L, V 5$, and V6 and an occasional RS pattern in V5 and V6 attributed to displaced transition of QRS complex

3. Absent $\mathrm{Q}$ waves in leads I, V5, and V6, but in the lead aVL, a narrow $Q$ wave may be present in the absence of myocardial pathology

4. $\mathrm{R}$ peak time $>60 \mathrm{~ms}$ in leads $\mathrm{V} 5$ and $\mathrm{V} 6$ but normal in leads V1, V2, and V3, when small initial $R$ waves can be discerned in the precordial leads

5. ST and T waves usually opposite in direction to QRS

LBBB affects ventricular repolarization resulting in STsegment deviation away from the QRS direction and in the setting of myocardial infarction, LBBB masks the ECG changes typical for MI such as ST/T wave changes and diminished Q wave.

Sgarbossa's ECG criteria was introduced to help clinician diagnosing myocardial infarction in the setting of LBBB, which then modified by Smith et al. (2012):

1. Concordant ST-segment elevation of $\geq 1 \mathrm{~mm}$ in the presence of a positive QRS complex

2. ST-segment depression of $\geq 1 \mathrm{~mm}$ in lead $\mathrm{V} 1, \mathrm{~V} 2$ or V3

3. Discordant ST-segment elevation with ST/S ratio of $\leq-$ 0.25 .

All measurements are made from the PR segment, and the ST segment is measured from the J-point. These criteria have an overall sensitivity of $91 \%$ and specificity of $90 \%$.

In the presented case, the patient came with typical angina symptoms for Acute Coronary Syndrome (ACS). Although the symptoms had occurred for 6 days before admission, the patient described his chest pain had gotten worse in the last 12 hours. The chest pain got worse with activity, was not relieved by rest, got longer in duration, and worse in quality, suggesting an acute destabilization (crescendo) of stable angina pectoris in ACS (Ibanez et al., 2018). Twelve-lead ECG at initial presentation concluded for LBBB, with seen pathologic $Q$ wave in inferior leads. Applying
Modified Sgarbossa's criteria for indicating MI in the setting of LBBB, 3 criteria were all negative in this initial ECG (Figure 1). There was no concordance ST Elevation, there was no ST Depression, and the ST/S ratio was -0.125 .

The presence of LBBB with pathologic $Q$ wave, in this case, suggested 2 diagnostic possibilities. First, it could be a STEMI masked behind a new LBBB suggesting a "STEMIequivalent" LBBB. The pathologic $\mathrm{Q}$ wave in inferior leads might also represent a $\geq 12$ hours new Inferior STEMI although this pattern is less likely with no ST-Elevation seen in inferior leads. Second, it indicated a Non-ST Elevation ACS (NSTEACS), either an Unstable Angina Pectoris or NSTEMI, accompanied with a chronic LBBB resulted from an Old Myocardial Infarct which may correlate to the history of the same chest pain the patient experienced 2 years ago, although there was no previous documented ECG available for this patient.

Based on the patients stable hemodynamic, history of similar typical angina pectoris, clear lungs on auscultation indicating no heart failure, a negative modified Sgarbossa's criteria at initial ECG, and a normal chest $\mathrm{x}$-ray and laboratory examination, the initial assessment of this patient were preferred as an NSTEACS with highly presumed chronic/old LBBB resulted from old myocardial infarction. The American College of Cardiology (ACC) proposed an algorithm where an echocardiogram and/or serum cardiac biomarkers should follow in a patient with MI and LBBB (new or old) and negative Sgarbossa criteria without heart failure or hemodynamic instability, whereas patients with heart failure or hemodynamic instability should undergo emergent percutaneous coronary intervention (PCI) or fibrinolysis regardless of Sgarbossa criteria (Beaty \& Park, 2020). Neither examination of cardiac biomarkers, echocardiogram, PCI, or fibrinolysis was available in our center. In this patient, either UAP or NSTEMI diagnosis could not be differentiated because of the unavailability of cardiac biomarkers examination. Our very limited settings require us to highly relying on clinical evaluation and serial ECG. The patient was decided for conservative therapy for NSTEACS with the plan of clinical, hemodynamic stability, and serial ECG evaluation.

Kusumoto et al. (2018) in AHA/ACC Guidelines stated that several cohort studies have demonstrated an association between LBBB in the development of coronary disease and heart failure. Damage to the left bundle branch of the HisPurkinje system resulted from ischemic or fibrotic can lead to LBBB in patients with past myocardial injury (Beaty \& Park, 2020). Other structural heart diseases that can cause chronic LBBB by damaging the His-Purkinje system and its branches are left ventricular hypertrophy from long-standing or uncontrolled hypertension, congestive heart failure resulting in ventricular remodeling, or valvular heart disease and cardiomyopathy resulting in dilatation, fibrosis, and advance cardiac remodeling. Conduction disorder due to degenerative disease causing Lenegre disease or sclerosis and calcification of cardiac skeleton (Lev Disease) may also lead to chronic LBBB (Cai et al., 2013; Neeland et al., 2012)

Anatomically, the left bundle branch is a large and diffuse structure. LBBB more commonly masks the ECG changes of the anterior or anteroseptal site with the involvement of a large infarction area, compared to any other location. In this case, a possibility of new inferior MI inducing LBBB was less likely because inferior and posterior infarction are less commonly inducing a new LBBB (Parekh et al., 2014). Moreover, instead of as a result of small or focal infarction area, the true new LBBB in AMI are often caused by either a very proximal coronary occlusion involving the septal 
perforating arteries supplying the proximal left bundle branch, or an extensive myocardial infarct involving a large portion of the distal conduction system including both fascicles to cause an acute injury. Thus, the true AMIassociated LBBB patients would usually be hemodynamically unstable and related to a very high mortality rate. (Cai et al., 2013; Neeland et al., 2012). Taking these into considerations, a new LBBB caused by STEMI is less likely in this case and the diagnosis tended to be NSTEACS with chronic LBBB caused by OMI.

In this case, the serial ECG was taken 12 hours after the initial ECG and the concordant ST-Elevation for $2 \mathrm{~mm}$ was found in precordial lead V3. Interestingly, the morphology of V3 was completely different in the second ECG compared to the first ECG initialing a transition lead. This morphology difference was highly suggestive of a variation of ECG lead placement rather than a sign of coronary occlusion "STEMI equivalent" in the new LBBB. This was also supported by the next serial ECGs recorded in this patient where no STElevation was found. Serial ECGs are useful in patients with suspected AMI and non-diagnostic initial ECG. Some factors need to be considered in taking serial ECG, and one of them is the variation of the position of precordial leads which can cause significant changes in ST-segment deviations. Marking the skin for lead location, using immovable adhesive electrodes, or using body-surface potential ECG mapping are suggested for alternatives, but the latter has not gained widespread use (Avsec et al., 2019; Kontos et al., 2011).

In regard to the ECG, while considering the clinical and hemodynamic stability, this patient was finally managed conservatively with an additional Fondaparinux regiment for 5 days. There was no other chest pain, the lungs remained clear and the hemodynamic remained stable throughout the hospital stays. The final clinical diagnosis was NSTEACS with OMI and chronic LBBB, although elective coronary angiography was highly suggested for this patient to evaluate the degree of the artery occlusion.

Several studies also supported our decision in the diagnostic and management of this case. Based on a study by Neeland et al. (2012), less than half of all patients with the diagnosis of suspected ACS and LBBB will finally be diagnosed with an AMI. Kontos et al. (2011) reported that MI was diagnosed in $29 \%$ of patients with LBBB with no significant difference in prevalence or size of MI among 3 groups: chronic LBBB, new LBBB, or presumed new LBBB. Chang et al. (2009) reported an even lower rate of AMI in patients with new or presumed new LBBB to be $7.3 \%$ and among those patients, only $19.2 \%$ had documented coronary disease by angiography, and $7.8 \%$ underwent coronary revascularization. In the more recent study, Pera et al. (2018) reported new LBBB was found in 3.3\% of suspected-STEMI patients who underwent $\mathrm{PCI}$ and a culprit lesion was present less frequently (54.2\%) in patients with new LBBB, compared to those without (83.3\%). Farre et al. (2015) reported in a study where only $35.8 \%$ of patients with LBBB underwent a primary PCI thrombotic coronary artery occlusion in contrast with $85.8 \%$ of patients with STEMI. These studies suggested that the prevalence of true new LBBB caused by AMI might be low in reality and on the contrary, signaling a high prevalence of false-positive catheterization laboratory activation. In fact, among STEMI patients with new LBBB, 44\% did not have culprit coronary occlusion on emergent angiography.

Patients with LBBB and MI may have a broad spectrum: a minority who has STEMI equivalent, patients with nonSTEMI equivalent who have myocardial necrosis but without a completely occluded vessel, or patients with acute decompensated heart failure with minor myocardial necrosis who mistakenly classified as having MI (Kontos et al., 2011). Thus, early reperfusion treatment may not be appropriate for all patients with new or presumed new LBBB and it is only the patients who have the real STEMI equivalent that emergent reperfusion treatment is truly beneficial.

The current recommendation of early reperfusion for all patients with new or presumed LBBB may have a great impact especially in the center where immediate access for early reperfusion is not readily available. Our center is located in a rural area with very limited diagnostic and therapeutic modalities and remote access to the higher facilitated center where PCI or fibrinolytic are available. In this case, false referral for early reperfusion may impose burdens for patients and healthcare providers during referral processes, unnecessary risk, and bigger healthcare expenses. False catheterization leads to increased risks of complications related to an invasive procedure, resulting in prolonged hospital stays and higher costs. On the other hand, unnecessary fibrinolytic therapy may impose patients for risks of bleeding and more severe complications in patients with older age, hypertension, and other comorbidities. Therefore, it is very important to distinguish whether the LBBB are categorized as new "STEMI equivalent" or old resulted from chronic process, and whether early reperfusion has more benefit in the patient with typical ACS because any decision therapy has a really big impact on patient outcome. This practice and application are even more important for a rural center where acuity in clinical judgment would benefit patient outcomes while preventing burdens and risks in early reperfusion option.

\section{CONCLUSION AND SUGGESTION}

Diagnosing AMI in the setting of LBBB propose a great challenge for clinician especially those in the rural center where diagnostic modalities are limited. Despite the recommendation of early reperfusion for patients with new or presumed new LBBB with AMI, only a minority are proven to have occluded artery signaling a high prevalence of false catheterization laboratory activation. Instead, chronic LBBB represents a bigger proportion which often resulted from ischemic and degenerative process from structural heart disease. Thorough anamnesis from present and past medical history, physical examination while considering hemodynamic stability, and careful serial ECG analyses would be the best approach in decision making and managing patients with LBBB and possible AMI in the setting of limited facilitation. The acuity in clinical judgment would benefit both patients and healthcare providers while avoiding unnecessary burdens, risks, and expenses resulted from false reperfusion therapy.

\section{ACKNOWLEDGEMENT}

We would like to express our gratitude to all those who have helped participate in this research, especially to the Health Polytechnic Ministry of Padang Padang, which have funded this research, through the DIPA fund of Health Polytechnic and the people of South Solok Regency who have carried out this research. 


\section{Funding Statement}

The authors did not receive support from any organization for the submitted work and No funding was received to assist with the preparation of this manuscript

\section{Conflict of Interest statement}

The author declares that there is no potential conflict of interest in relation to the authorship and publication of this article.

\section{REFERENCES}

Avsec, M., Fister, M., Noč, M., \& Radsel, P. (2019). Alternating bundle-branch block in acute coronary syndrome. Cor et Vasa, 61(1), $68-71$. https://doi.org/10.1016/i.crvasa.2017.12.013

Beaty, B., \& Park, D. (2020). Diagnosis of myocardial infarction in a patient with left bundle branch block and negative sgarbossa criteria. Journal of Osteopathic Medicine, 120(10), 655-659. https://doi.org/10.7556/jaoa.2020.107

Cai, Q., Mehta, N., Sgarbossa, E. B., Pinski, S. L., Wagner, G. S., Califf, R. M., \& Barbagelata, A. (2013). The left bundle-branch block puzzle in the 2013 ST-elevation myocardial infarction guideline: From falsely declaring emergency to denying reperfusion in a high-risk population. Are the Sgarbossa Criteria ready for prime time? American Heart Journal, 166(3), 409-413. https://doi.org/10.1016/j.ahj.2013.03.032

Chang, A. M., Shofer, F. S., Tabas, J. A., Magid, D. J., McCusker, C. M., \& Hollander, J. E. (2009). Lack of association between left bundle-branch block and acute myocardial infarction in symptomatic ED patients. The American Journal of Emergency Medicine, 278), 916-921. https://doi.org/10.1016/j.ajem.2008.07.007

Farré, N., Mercè, J., Camprubí, M., Mohandes, M., Guarinos, J., Fernández, F., Oliva, X., \& Bardají, A. (2015). Prevalence and outcome of patients with left bundle branch block and suspected acute myocardial infarction. International Journal of Cardiology, 182, 164-165. https://doi.org/10.1016/j.ijcard.2014.12.123

Ibanez, B., James, S., Agewall, S., Antunes, M. J., Bucciarelli-Ducci, C., Bueno, H., Caforio, A. L. P., Crea, F., Goudevenos, J. A., Halvorsen, S., Hindricks, G., Kastrati, A., Lenzen, M. J., Prescott, E., Roffi, M., Valgimigli, M., Varenhorst, C., Vranckx, P., Widimský, P., ... Gale, C. P. (2018). 2017 ESC Guidelines for the management of acute myocardial infarction in patients presenting with ST-segment elevation. European Heart Journal, 392), 119-177. https://doi.org/10.1093/eurheartj/ehx393

Kontos, M. C., Aziz, H. A., Chau, V. Q., Roberts, C. S., Ornato, J. P., \& Vetrovec, G. W. (2011). Outcomes in patients with chronicity of left bundle-branch block with possible acute myocardial infarction. American Heart Journal, 161(4), 698-704. https://doi.org/10.1016/j.ahj.2011.01.008

Kusumoto, F. M., Schoenfeld, M. H., Barrett, C., Edgerton, J. R., Ellenbogen, K. A., Gold, M. R., Goldschlager, N. F., Hamilton, R. M., Joglar, J. A., Kim, R. J., Lee, R., Marine, J. E., McLeod, C. J., Oken, K. R., Patton, K. K., Pellegrini, C. N., Selzman, K. A., Thompson, A., \& Varosy, P. D. (2019). 2018 acc/aha/hrs guideline on the evaluation and management of patients with bradycardia and cardiac conduction delay: A report of the american college of cardiology/american heart association task force on clinical practice guidelines and the heart rhythm society. Circulation, 140(8). https://doi.org/10.1161/CIR.0000000000000628

Neeland, I. J., Kontos, M. C., \& de Lemos, J. A. (2012). Evolving considerations in the management of patients with left bundle branch block and suspected myocardial infarction.Journal of the American College of Cardiology, 60(2), https://doi.org/10.1016/j.jacc.2012.02.054 96-105.

Parekh, P., Agrawal, N., Vasavada, A., \& Vinchurkar, M. (2014). LBBB masking the ECG changes of inferior wall infarction: A caution to be vigilant. Case Reports, 2014, bcr2014205095. https://doi.org/10.1136/bcr-2014-205095

Pera, V. K., Larson, D. M., Sharkey, S. W., Garberich, R. F., Solie, C. J., Wang, Y. L., Traverse, J. H., Poulose, A. K., \& Henry, T. D. (2018). New or presumed new left bundle branch block in patients with suspected ST-elevation myocardial infarction. European Heart Journal: Acute Cardiovascular Care, 73 ), 208-217. https://doi.org/10.1177/2048872617691508

Smith, S. W., Dodd, K. W., Henry, T. D., Dvorak, D. M., \& Pearce, L. A. (2012). Diagnosis of ST-elevation myocardial infarction in the presence of left bundle branch block with the STelevation to S-wave ratio in a modified Sgarbossa rule. Annals of Emergency Medicine, 606), 766-776. https://doi.org/10.1016/j.annemergmed.2012.07.119

Wahab, M. A. K. A. (2017). Ischemia modified albumin (Ima) in acute coronary syndrome (Acs) and left bundle branch block (Lbbb). Does it make the difference? The Egyptian Heart Journal, 69(3), 183-190. 
